\title{
Estrogen, estrogen receptors and rupture of brain aneurysms: brief review of the literature
}

\author{
Sandy Zuleica Navas-Marrugo, Hernando Raphael Alvis-Miranda, \\ Juan Jose Gutierrez-Paternina, Gabriel Alcala-Cerra, Luis Rafael \\ Moscote-Salazar
}

Universidad de Cartagena

\begin{abstract}
Estrogen, is involved in much of the life women lives, having great importance in many functions of the female body including the definition of secondary sexual characters, but also has been linked as protective factor regard to catastrophic events such as cerebral aneurysms, probably related to estrogen deficiency and described high incidence in menopausal women. It is known the effects of estrogen on vascular physiology and pathophysiologic mechanisms with potential therapeutic implications. We present a brief review focused in the role of estrogens and the rupture of cerebral aneurysms.
\end{abstract}

Key words: estrogen, cerebral aneurysms, deficiency, menopause.

\section{Introduction}

About $20 \%$ of strokes are hemorrhagic events, from these subarachnoid hemorrhage (SAH) accounts for $10 \%$ the remaining half corresponds tointracerebral hemorrhage. It is known that cerebral aneurysms are more common in women; numerous studies suggest the involvement of hormones in its pathogenesis. Studies have shown that oral contraceptives and hormone replacement therapy may be protective against aneurysmal subarachnoid hemorrhage suggesting that cyclical hormonal fluctuations in the context of physiological menses are associated with cerebral vascular instability and then the formation of cerebral aneurysms. Most causes SAH are ruptured berry aneurysms. Other causes include trauma, arteriovenous malformations, vasculitis, intracranial arterial dissections, amyloid angiopathy, bleeding diathesis, and illegal psychoactive substances such as cocaine and amphetamines. The aim of this work is to review the current kwnoledge regard aneurysms of the cerebral vasculature and their relationship with estrogen.

\section{Subarachnoid hemorrhage}

$\mathrm{SAH}$ is one of the most catastrophic intracranial events that may suffer an individual to whichneurosurgeons are routinely faced. It is defined as the occupation or invasion of blood in the subarachnoid space, where normally circulates the 
cerebrospinal fluid (CSF). Aneurysmal rupture leads the patient to a critical state, with high probability of mortality. The care and management of patients with subarachnoid haemorrhage is vital to the successful development, as well as a multidisciplinary approach where participating neurosurgeons and endovascular neurosurgeons should be experienced in the microsurgical management of cerebral aneurysms.

\section{Epidemiology}

It is located within the pathologies that inevitably must handle any physician, affecting 6-10 people per 100,000 per year, although this range can varie (depending on the studies), in Finland, there have been incidents that reach 20/100.000 patients/ Year. (2)

The most common age of onset is around 55 years, increasing incidence with increasing age. It is also more common in women. (2)According to retrospective studies, among all patients who attend the emergency department with headache, subarachnoid hemorrhage is the cause in $1 \%$ of cases. Considering only patients with the worst headache of their lives and normal physical examination, this figure rises to $12 \%$. This ratio increases again taking into account the patients with abnormal physical examination, reaching $25 \%$. (3)

It is a severe disease with a mortality rate of approximately 20 to $40 \%$ of inpatients plus 8 $15 \%$ mortality in the first minutes or hours, in the prehospital phase. (3)

\section{Etiology}

This process can be a pathological entity subsequent to trauma or occur spontaneously. Being the main cause of the latter rupture of an aneurysm in the brain stem ( $85 \%$ of cases) (4).

The causes of spontaneous SAH are saccular aneurysm rupture (85\%), ruptured arteriovenous malformation ( $8 \%-10 \%)$, unknown etiology (10\%) and other rare (hypertension, arteritis, tumors, cardiac myxoma, and septic aneurysms). (4)

Cerebral aneurysms are saccular dilatations of the arterial wall that are located at the forks of the basal subarachnoid brain arteries or near the circle of Willis. They originate from sites where there is a birth defect of the middle layer and the inner elastic membrane.

Several modifiable risk factors have been identified in relation to the HSA.

\section{Clinical features}

The signs and symptoms of this disease are of sudden onset, in an individual who usually had no previous neurological disorders.

The onset of symptoms is often preceded by intense physical activity or sexual activity, although it was noted that in a series of 500 patients with subarachnoid hemorrhage, in $34 \%$ of cases developed during stressful activities and $12 \%$ were produced during sleep.

Headache, which is the most common symptom, is presented as sudden onset and intense, usually referred as the worst. Often is accompanied by nausea and vomiting. They can have any location, can be localized or generalized, may be mild and resolve spontaneously, may be relieved or nonrelieved by non-narcotic analgesics. Facing the 
DOI: 10.2478/romneu-2014-0039

first or worst headache and an unusual headache in a patient with an established pattern of pain, SAH should be suspected until proven otherwise.

\section{Estrogen}

Estrogens are female sex hormones that participate in a variety of actions on different tissues. Among others, stimulate the development of female secondary sex characteristics, are involved in the characterization of the female physiognomy, adapting fatty deposits at the hips, thighs and development of internal and external genitalia.

It acts on endometrial proliferation and growth of the uterus during the menstrual cycle in the cornification of the superficial cells of the vaginal mucosa, increases the secretion of cervical mucus to have the crossing of the sperm through the cervical mucus.

Estrogens alter serum lipid levels, the fibrinolytic system and coagulation, antioxidant systems, and the production of vasoactive molecules, influencing the development of vascular disease. (1). However, direct exert pleiotropic effects on endothelial cells, collagen vascular smooth muscle constituent, and a potent vasodilator called nitric oxide (NO), thus contributing, together with other substances, the normal homeostatic process that allows endothelial remodeling arteries to maintain a constant diameter of the lumen, and therefore laminar blood flow strong enough for proper irrigation to distant structures. Thanks to this, with any noxious event affecting the vasculature, endothelial cells have the ability to counteract all processes efficiently generated.
Atherosclerosis, which is a late consequence of all inflammatory and oxidative processes generated in endothelial cells has been associated with the formation, growth and ruptured aneurysms in any location. Although not yet demonstrated a direct causal link between the two mechanisms, histopathological findings of the atheromatous plaque have been identified in some samples of intracranial aneurysm wall, a fact that has allowed these inflammatory phenomena gain importance, taking into account that could be a therapeutic target in the pathogenesis of aneurysmal cerebrovascular disease.

The sequence of inflammatory phenomena at endothelial level can be described as follows: endothelial dysfunction, vasoconstriction, leukocyte and monocyte adhesion, increased vascular permeability, monocyte migration and leukocytes, programmed cell death, release of proteolytic enzymes. It is not known with certainty how the metabolic pathway of estrogen reduce vascular tone, by direct action of NO and prostacyclin and inhibit monocyte adhesion to the endothelial wall by reducing plasma levels of the molecules VCAM-1 and MCP -1. The latter is probably due to inhibition in the expression of genes that produce such molecules, after intercepting the nuclear transcription factor NF- $\kappa \beta$. Furthermore, it is suspected that it is also responsible for the inhibition of MMP-2 and MMP-9, two major metalloproteinases that destroy the collagen and elastin of smooth muscle located in the arterial wall.

All these actions are performed through the interaction of the hormone with its specific 
receptor. There are four different types: ER- $\alpha$, ER- $\beta$ (which are activated by ligands) IPG (also called GPR 30, which is coupled to protein G), and ER-X, all available at level of the endothelial cells of the cerebral vasculature.

Estrogens are able to directly influence the fallopian tube, muscle, breast cancer, pituitary gland, bone, smooth muscle, ion transport mechanisms, among others.

In the uterus, increases spontaneous contractions expanding the excitation of oxytocin in the tubes, increases in size, and breast in turn, lead to a proliferation of ducts. In the hypothalamus, are responsible for the secretion of LHRH and dopamine, through which reduced gonadotropin levels also stimulate the production of prolactin, however limit their actions on the breast, also favor the presence of receptors for FSH the hair and become more sensitive to their stimulating hormone pituitary hypothalamic LHRH.

Bones, provide mineralization, regularized long bone growth, cartilage conjunction closed and therefore cease growing. On the vascular system, estrogens may align or alter the ionic fluxes, receivers and reproduction property of vascular smooth muscle cells, and also regulate the release of endothelium-derived vasoactive factors. Thus, estrogen increases competition gear dependent vasodilator $\mathrm{NO}$ and prostacyclin, reduce the effectiveness of vasoconstrictors mechanisms, namely prostaglandins, RAAS (renin angiotensin aldosterone system), endothelin-1 (there is conflicting evidence on this ), involved in ion channel $\mathrm{K}+$ and $\mathrm{Ca} 2+$ in the cells of the vascular smooth muscle (VSMC), prompting hyperpolarization and decreasing its contraction, worth noting the actions of estrogen on the lipid profile, they are able to reduce the plasma concentration of LDL and increase HDL.

Estrogens are hormones quintessential female puberty as they provide the characteristic distribution of body fat, which distinguish the female physiognomy predominantly around the hips and thighs of the male, which is mainly abdominal.

It is seen that the activity of these hormones are present in most of women's lives, play an essential role in the biology of the vascular system and its mechanisms may represent beneficial results, however, is questioned their involvement in the pathophysiology of intracranial aneurysms (1).

The fact that cerebral aneurysms are more common in women is not new. Epidemiological data indicate a high incidence of cerebral aneurysms in postmenopausal women (2-3). Numerous studies on the involvement of hormonal factors had treated the pathogenesis of aneurysms, is known the great participation of estrogen at different stages of the life of the woman, the great interaction between them and the vascular biology and although these interactions charities are largely still considers its involvement in the pathophysiology of cerebral aneurysms and catastrophic health of women. (4) (5) (1)

\section{Endothelial and hormonal physiology}

The endothelium is a layer of tissue which covers all the vessels of the body. Endothelial cells have a selective permeability, having non-thrombogenic properties, substantial 
DOI: 10.2478/romneu-2014-0039

metabolic activity and the ability to produce various vasoactive substances. In this way, the most important properties that can be identified on the endothelium are:

- Selective permeability: Forms a highly selective permeability barrier, regulating the flow of nutrients, bioloigcamente active molecules and blood cells. (7).

- Maintenance balance between thrombosis and fibrinolysis.

\section{Progression of the disease}

Cerebrovascular disease is favored by oxidative stress, increases inflammation, and may trigger processes that contribute to the formation and subsequent rupture of an aneurysm, as direct endothelial injury, phenotypic change of smooth muscle cells to a phenotype of inflammatory and Finally the radical apoptosis. Free radicals are also able to activate the matrix metalloproteinases getting vessel remodeling and decomposition. These radicals in turn involved in all mechanisms responsible for cerebral aneurysm, such as atherosclerosis, hemodynamic stress and hypertensive pathology that makes it through the mechanism of peroxidation. Thus, some preliminary studies involving targeted therapies to oxidative stress as a beneficial therapeutic option for treating cerebral aneurysms future, however, recent studies point to define the role of free radicals in the formation and rupture of a cerebral aneurysm. (6)

\section{Estrogen and brain aneurysm formation}

The hypothesis regarding the alterations in hormone levels can influence the $\mathrm{SAH}$ is gaining momentum rapidly. Specifically, the concept that post-menopausal women are more susceptible to the condition has convinced many of the protective roles of estrogen and progesterone (7).

The task of the estrogen is very important for vascular biology; its beneficial effects are well known but not participating in the pathogenesis of cerebral aneurysms (1). Progression is associated with endothelial damage (2) to inflammation, impaired extracellular matrix and extracellular wall apoptosis, also in this process have been described as statins growth initiation mechanism and the aneurysm. (8)

Variations of estrogen level have been associated with the development and progression and rupture of cerebral aneurysms, which could explain the gender gap regarding the higher incidence found in women. In a case-control study which interviewed 4,682 women, mostly observed premature menopause ( $<40$ years) associated with aneurysm formation, which suggests that reducing estrogen may be premature in training a cerebral aneurysm. This in turn may lead to a risk factor to explain the pathophysiology of cerebral aneurysms and for long term therapies.

The growth in the incidence of cerebral aneurysms in postmenopausal women can relate diminished circulating estrogen levels. In a rat model, for example, which induced the formation of the aneurysm, the researchers found that in oophorectomized models increased incidence of cerebral aneurysms (9)

Involving the renin-angiotensin (Ang)aldosterone in the pathology of vascular disease has also been described. However 
incommunicado mineralocorticoid receptor protects against vascular diseases, their influence on brain aneurysms is unclear. In a study used female rats which were induced by renal hypertension, increased hemodynamic stress, and they were subjected to insufficient estrogen for 3 months with the mineralocorticoid receptor blocker eplerenone (30 or $100 \mathrm{mg} / \mathrm{kg}$ per day) or vehicle (vehicle control) was observed as eplerenone reduced the incidence of cerebral aneurysms and saline intake without reducing blood pressure. On the wall of the aneurysm, increased production of Ang II and nitrotyrosine. MRNA levels of Ang-converting enzyme 1 and NADPH (10)

Gender inequalities in the progression of aneurysmal subarachnoid hemorrhage (SAH) are discussed, and the possible influence of estradiol on vasodilation is not well defined. (11) Large number of studies suggests that hormones are important in the pathogenesis of an aneurysm. Estrogen raises the normal physiological vascular endothelial employment, and waves during the menstrual cycle as well as drops significantly at menopause, which would explain such relationship (5).

\section{Neuroprotective factors}

Evidence for a protective role of estradiol in neurodegenerative diseases has increased steadily over the past decade, although the mechanisms of action and participation of estrogen receptors (ER) showed a complex result. The protective effects of estrogens take place partly through pathways involving activation of canonical ER, which is constitutively expressed in many brain regions and is capable of initiating transcription of genes after specifically bind estradiol. Furthermore, non-genomic pathways (or alternative), involving extranuclear RE respond to physiological concentration of estrogen to induce neuroprotection. Often, rapid activation of intracellular signaling, such as mitogen activated protein kinase (MAPK) and phosphatidylinositol 3-kinase (PI3K) underlying estrogen-induced neuroprotection alternative activation of specific binding sites in the plasma membrane. Although the molecular characteristics of these unconventional ERs are still largely unknown, the conventional wisdom holds that the plasma membrane ER (MER) arises from or relates to, classical nuclear ER. This article reviews some of the latest evidence that reveals the importance of alternative mechanisms for estrogen-dependent neuroprotection. Special attention to models of cellular toxicity of betaamyloid in classical and alternative pathways activated by estrogens seem to exist to orchestrate neuroprotection. (12).

\section{Conclusions}

Knowledge of the cellular mechanisms involved in the possible involvement of estrogen and cerebral aneurysm formation will be an interesting line of research that may offer additional treatment alternatives for patients with incidental aneurysms or in patients at risk for brain aneurysms.

\section{Correspondence}

Dr. Luis Rafael Moscote-Salazar, University of Cartagena, Cartagena de Indias, Colombia, Southamerica.E-mail:mineurocirujano@aol.com 
DOI: 10.2478/romneu-2014-0039

\section{References}

1. Jamous MA, Nagahiro S, Kitazato KT, Satomi J, Satoh $\mathrm{K}$. Role of estrogen deficiency in the formation and progression of cerebral aneurysms. Part I: experimental study of the effect of oophorectomy in rats. Journal of neurosurgery [Internet]. 2005 Dec [cited 2013 May 26], 103 (6): 1046-51. Available from: http://www.ncbi.nlm.nih.gov/pubmed/16381191

2. Tamura T, Jamous MA, Kitazato KT, Yagi K, Tada Y, Uno $M$, et al. Endothelial damage due to impaired nitric oxide bioavailability triggers cerebral aneurysm formation in female rats. Journal of hypertension [Internet]. 2009 Jun [cited 2013 May 25], 27 (6) :1284-92. Available from: http://www.ncbi.nlm.nih.gov/pubmed/19307983

3. Ding C, Toll V, Ouyang B, Chen M. Younger age of menopause in women with cerebral aneurysms. Neurointerv J Surg [Internet]. 2012 June 13 [cited 2013 May 15] Available from: http://www.ncbi.nlm.nih.gov/pubmed/22700728

4. Golledge J, Biros E, Warrington N, Jones GT, Cooper M, Van Rij AM, et al. A population-based study of polymorphisms in genes related to sex hormones and abdominal aortic aneurysm. Eur J Hum Genet [Internet]. 2011 Mar [cited 2013 April 11], 19 (3): 363-6. Available from:

http://www.pubmedcentral.nih.gov/articlerender.fcgi?ar tid $=3062004 \&$ tool $=$ pmcentrez\&rendertype $=$ abstract

5. Chen M, Ouyang B, Goldstein-Smith L, Feldman L. Oral contraceptive and hormone replacement therapy in women with cerebral aneurysms. Journal of neurointerventional surgery [Internet]. 2011 Jun [cited 2013 May 25], 3 (2): 163-6. Available from: http://www.ncbi.nlm.nih.gov/pubmed/21990811

6. Starke RM, Chalouhi N, Ali MS, Jabbour PM, Tjoumakaris SI, Gonzalez LF, et al. The Role of Oxidative Stress in Cerebral Aneurysm Formation and Rupture. Current neurovascular research [Internet]. 2013 May 24 [cited 2013 Jun 3] Available from: http://www.ncbi.nlm.nih.gov/pubmed/23713738
7. Young AMH, Karri SK, Ogilvy CS. Exploring the use of estrogen \& progesterone replacement therapy in subarachnoid hemorrhage. Current drug safety [Internet]. 2012 Jun [cited 2013 May 25], 7 (3) :202-6. Available from: http://www.ncbi.nlm.nih.gov/pubmed/22950381 8. Tada Y, Kitazato KT, Yagi K, Shimada K, Matsushita N, Kinouchi T, et al. Statins Promote the growth of experimentally induced cerebral aneurysms in estrogendeficient rats. Stroke, a journal of cerebral circulation [Internet]. 2011 Jun [cited 2013 May 25], 42 (8) :2286-93. Available from: http://www.ncbi.nlm.nih.gov/pubmed/21737796 9. Jamous MA, Nagahiro S, Kitazato KT, Tamura T, Kuwayama K, Satoh K. Role of estrogen deficiency in the formation and progression of cerebral aneurysms. Part II: experimental study of the effects of hormone replacement therapy in rats. Journal of neurosurgery [Internet]. 2005 Dec [cited 2013 May 26], 103 (6) :1052-7. Available from: http://www.ncbi.nlm.nih.gov/pubmed/16381192

10. CL Lin, Shih HC, Dumont AS, Kassell NF, Lieu AS, Su YF, et al. The effect of 17beta-estradiol in attenuating experimental subarachnoid hemorrhage-induced cerebral vasospasm. Journal of neurosurgery [Internet]. 2006 Feb [cited 2013 May 26], 104 (2) :298-304. Available from: http://www.ncbi.nlm.nih.gov/pubmed/16509505 11. Yang SH, He Z, Wu SS, He YJ, Cutright J, Millard WJ, et al. 17-beta estradiol can reduce secondary ischemic damage and mortality of subarachnoid hemorrhage. Journal of cerebral blood flow and metabolism: official journal of the International Society of Cerebral Blood Flow and Metabolism [Internet]. $2001 \mathrm{Feb}$ [cited 2013 May 26], 21 (2): 174-81. Available from: http://www.ncbi.nlm.nih.gov/pubmed/11176283

12. Marin R, Guerra B, Alonso R, Ramírez CM, Diaz M. Estrogen activates classical and alternative mechanisms flag to orchestrate neuroprotection. Current neurovascular research [Internet]. 2005 Oct [cited 2013 May 26] 2 (4): 287-301. Available from: http://www.ncbi.nlm.nih.gov/pubmed/16181121 